\title{
Civilisations
}

Revue internationale d'anthropologie et de sciences

humaines

$64 \mid 2015$

Enquêter en terrains difficiles

\section{Enquêter en prison auprès de femmes impliquées dans des violences sexuelles sur mineurs}

\section{Myriam Joël}

\section{(2) OpenEdition}

\section{Journals}

Édition électronique

URL : http://journals.openedition.org/civilisations/3861

DOI : $10.4000 /$ civilisations.3861

ISSN : 2032-0442

\section{Éditeur}

Institut de sociologie de l'Université Libre de Bruxelles

\section{Édition imprimée}

Date de publication : 22 décembre 2015

Pagination : $69-79$

ISSN : 0009-8140

\section{Référence électronique}

Myriam Joël, «Enquêter en prison auprès de femmes impliquées dans des violences sexuelles sur mineurs ", Civilisations [En ligne], 64 | 2015, mis en ligne le 30 décembre 2018, consulté le 04 janvier 2020. URL : http://journals.openedition.org/civilisations/3861 ; DOI : 10.4000/civilisations.3861 


\title{
Enquêter en prison auprès de femmes impliquées dans des violences sexuelles sur mineurs
}

\author{
Myriam JOËL
}

Résumé : Si les travaux sociologiques sur les femmes violentes font figure d'exception, les études sur les femmes auteures de violences sexuelles sur mineurs sont quant à elles inexistantes. L'article propose un point de départ à la réflexion au prisme d'une interrogation d'ordre méthodologique: quelles difficultés rencontre le sociologue travaillant sur un tel objet? La première concerne l'accès aux enquêtées en prison. Si la réalisation d'entretiens répétés permet d'établir une solide relation de confiance propre à favoriser les confidences les plus terribles, les autres acteurs de la détention manifestent des réticences et de la réprobation face à l'attention qui leur est portée. Le second obstacle renvoie à la sensation de souillure associée au recueil de données, le sociologue s'exposant à un risque de compromission éthique. A la contamination symbolique peut cependant s'adjoindre un curieux sentiment d'allégresse à l'écoute de récits épouvantables mais féconds pour la recherche. La consubstantialité de la sociologie et de l'engagement moral pour le "bien " des acteurs sociaux a pour conséquence qu'il est sociologiquement incorrect d'évoquer cette forme de jubilation. Enfin si la réalisation d'une telle enquête captive, la diffusion des résultats tend à réactiver le registre "chaud» de la révolte et de l'indignation.

Mots-clés : indicible, femmes, mineurs, prison, violences sexuelles.

\begin{abstract}
Sociological investigations on violent women are exceptional and studies on female sexual offenders are non-existent. This article proposes a starting point of reflection by exploring methodological aspects: which difficulties sociologist encounters by working on such an objet? The first one regards the access to respondents in prison. Repeated interviews enable a solid and trust relationship which promotes the sharing of most terrible secrets. However, other actors of detention show reluctance and disapproval relative to this attention. The second obstacle relates to the feeling of defilement, which is associated with data collection. Indeed sociologist exposes him-herself to an ethical compromise risk. But besides this symbolic contamination, he-she can also experience a curious feeling of glee when he-she is listening to frightful but fruitful stories for his-her research. Thus we can point out a consequence of the consubstantiality of sociology and moral commitment for social actors' welfare: it is sociologically incorrect to raise this form of jubilation. Finally, such a survey captivates, but transmission of results tends to reactivate the "hot" registry of revolt and indignation.
\end{abstract}

Keywords: unspeakable, women, minors, prison, sexual violence. 


\section{Introduction}

Si les travaux sociologiques sur les femmes violentes font figure d'exception, les études sur les femmes auteures de violences sexuelles sont quant à elles inexistantes. Il existe une étroite relation entre ce vide sociologique et l'occultation telle qu'elle caractérise également l'espace public, les médias concourant à alimenter l'image préconstruite d'une violence spécifiquement masculine. La question des violences sexuelles envers les enfants est paroxystique de cette situation. Présentées comme complices, soumises, contraintes ou manipulées par leur conjoint, les femmes impliquées dans ce type d'agression se voient invariablement assigner un rôle passif dans les faits qu'on leur reproche. Se mêlent ainsi un processus de psychologisation inscrivant cette violence dans une histoire familiale lui donnant sens, et une inclusion de cette violence à l'intérieur d'une domination masculine lui conférant un caractère de subordination (Cardi et Pruvost, 2011). La partition femme victime/homme agresseur irrigue à ce point les représentations collectives qu'il est difficile - pour les sociologues y compris ${ }^{1}$ - de concevoir qu'une femme puisse être l'auteure de telles conduites.

On peut faire l'hypothèse de deux grands facteurs à l'origine de ce problème de dicibilité sociale. Etayé par l'argument de la rareté de ce type d'infractions ${ }^{2}$, le premier renvoie au système de représentations circonscrivant l'acte sexuel au coït et donc l'agression sexuelle au viol. Dépourvues de pénis, les femmes endossent laborieusement le rôle d'agresseur sexuel dans l'imaginaire collectif. Les stéréotypes de genre contribuent d'autre part à faire de cette question un non-objet, la douceur de la « nature » féminine s'avérant incompatible avec la prise d'initiative d'une activité sexuelle à caractère coercitif. Exercée contre des enfants, cette violence cristallise la double transgression associée à la déviance féminine : une transgression légale à laquelle vient se surimposer une transgression de genre (Cardi, 2008), les femmes en question étant en rupture totale avec les rôles sociaux de care qui leur sont assignés. Il en résulte une indicibilité sociale dont l'indice le plus manifeste est l'absence d'intérêt des sociologues travaillant sur la prison, lieu d'accueil des personnes accusées de ce type d'infraction. Plusieurs études se sont intéressées au traitement des hommes détenus auteurs de violences sexuelles sur mineurs (Faure et al., 1996 ; Gaillard, 2008 ; Brie, 2014) mais la situation chez les femmes n'a en revanche été que peu, voire pas du tout, explorée.

L'article vise à proposer un point de départ au prisme d'une interrogation d'ordre méthodologique : comment enquêter sur un terrain auquel le caractère à la fois horsnorme, indicible et troublant confère une indéniable difficulté ? Comment le sociologue $\mathrm{y}$ accède-t-il? Comment gère-t-il les données recueillies ? Comment exploite-t-il les résultats obtenus? Que dit une telle enquête du métier de sociologue ? Pour tenter de répondre à ces questions, je livrerai une réflexion à partir de ma recherche qualitative sur la sexualité en prison de femmes, au cours de laquelle je menai un suivi longitudinal

1 Ces difficultés à penser la violence féminine se retrouvent dans les travaux sur les mères maltraitantes, les auteurs les décrivant couramment comme des femmes « en détresse ».

2 La criminologue Claudia Melcher (2002) souligne néanmoins que les résultats des études divergent fortement; et l'historienne Fabienne Giuliani (2011) indique que les archives lacunaires laissent supposer l'existence d'un phénomène bien présent tout au long du siècle. 
qualitatif de quatre-vingt détenues durant un an et des entretiens individuels et collectifs avec soixante-quinze surveillantes, gradés, membres du personnel de direction, travailleurs sociaux, soignants, intervenants extérieurs et magistrats. Pour épineuse qu'elle soit, la question des violences sexuelles sur mineurs irrigua l'étude, tant sur le terrain proprement dit qu'en aval, au cours de la valorisation de la recherche. Dans une première partie, nous nous intéresserons aux difficultés d'accès aux femmes concernées. Nous porterons ensuite notre attention sur la sensation de souillure associée au recueil de données. Puis nous nous interrogerons sur le déconcertant sentiment d'allégresse éprouvé à l'écoute de récits épouvantables mais féconds pour la recherche. Enfin nous nous pencherons sur l'exploitation de données sensationnelles venant indubitablement troubler l'ordre social.

\section{Réticences et indignation au sein de la détention féminine}

L'accès aux établissements pénitentiaires est généralement décrit par les sociologues comme un parcours semé d'embûches. Si l'obtention des autorisations s'avère en effet laborieuse pour le chercheur non familier du milieu carcéral et des rouages de l'Administration pénitentiaire (AP), elle est relativement aisée à qui s'est déjà constitué un réseau et surtout qui s'est gardé dans ses travaux d'un positionnement idéologique mettant en accusation l'AP. Echaudés par le discours public fantasmé sur la prison, les représentants de l'AP restreignent en effet l'accès au terrain par crainte de la péjoration sociale qu'ils subissent dans les médias et dont de nombreux travaux scientifiques se font le relais (Benguigui et al., 1994). En dépit du caractère polémique de mon objet d'étude, la connaissance par mes interlocuteurs institutionnels de ma prise de distance avec tout engagement militant suffit donc à neutraliser leurs doutes quant à mes intentions. Je ne rencontrai aucune réaction d'hostilité ou de crainte à l'annonce de mon projet de recherche. Bien au contraire, ma démarche fut accueillie avec enthousiasme et l'on me félicita même de m'intéresser à un sujet sur lequel existait aussi peu d'informations. J'obtins ainsi plus d'autorisations d'accès que je ne pouvais en utiliser. Dans l'enceinte de la détention en revanche, je fus confrontée à deux obstacles.

Le premier concerne les réticences exprimées par les agents pénitentiaires dans le recrutement de mes enquêtées. Lorsque je débutai ma recherche, j'étais alors âgée de vingt-trois ans mais j'en paraissais beaucoup moins, notamment parce que je m'efforçais de renvoyer l'image d'une étudiante candide et inoffensive ; le statut d'étudiant se révélant favorable au travail d'enquête (Bizeul, 1998). Mes relations avec les responsables de quartier ou de bâtiment se construisirent dès lors sur un mélange de familiarité et de protection à mon égard. Plusieurs se montrèrent inquiets de me savoir confrontée à des récits susceptibles de me déstabiliser, ces craintes s'exprimant avec acuité dans le cas des détenues impliquées dans des violences sexuelles sur mineurs. Non seulement on m'enjoignit de ne pas hésiter à interrompre celles qui s'aviseraient de me raconter « des horreurs », mais on tenta même de me dissuader de les rencontrer en me mettant en garde vis-à-vis des déclarations " perverses » et des propositions équivoques qu'on risquait de me faire.

Le filtrage dans le recrutement de mes enquêtées releva également d'une autre logique qu'est le biais de représentativité : préoccupés par l'extrapolation et la généralisation des résultats de l'étude, les agents pénitentiaires sont réticents à ce que 
le chercheur rencontre des personnes détenues non représentatives de la population incarcérée (Cliquennois, 2006). Je ressentis ainsi de leur part des réserves à ce que je suive des détenues dont le comportement attestait d'un écart important avec les modèles de conduite traditionnellement assignés aux femmes. Si cette tentative de contrôle s'observa dans le cas des détenues obscènes et maltraitantes, elle s'intensifia avec les femmes impliquées dans des violences sexuelles sur mineurs. Je décelais dans les discours désapprobateurs des agents la crainte que ces rencontres ne m'amènent à nourrir des représentations négatives à l'endroit de la population féminine incarcérée dans son ensemble, celles-ci risquant alors de se projeter sur eux au regard du caractère contagieux du stigmate. Leur inquiétude se manifestait d'ailleurs en creux de leur empressement à me mettre en contact avec certaines détenues. La fierté avec laquelle ils me décrivaient leurs capitaux ne laisse aucun doute quant à la relation d'interdépendance unissant l'image des personnes détenues à celle des agents qui en ont la charge.

Ma venue régulière dans les établissements me permit de surmonter ces difficultés. $\mathrm{Au}$ fil du temps, les agents pénitentiaires comprirent d'une part que j'étais dans l'optique de rencontrer des détenues au profil différent; et constatèrent d'autre part que je ne semblais point traumatisée par ce qu'on me racontait puisque je participais même aux plaisanteries macabres qui alimentent le quotidien de la détention. Avec la population incarcérée en revanche, je me heurtai à une ferme réprobation qui compliqua la réalisation du suivi. Les entretiens se déroulaient souvent au cœur de la détention, dans un bureau pourvu d'une vitre transparente devant lequel passaient les détenues. Dès lors, toutes me voyaient rire ou serrer la main des femmes impliquées dans des violences sexuelles sur mineur, et pouvaient estimer le temps que nous passions ensemble, celui-ci excédant souvent plusieurs heures. Or l'on observe au sein de la détention féminine un processus d'exclusion à l'endroit de ces détenues, par lequel on les assigne à une position hors-genre au regard de leur transgression des rôles sociaux traditionnels de care.

Majoritairement issues des groupes populaires où prédomine une forte division sexuelle du travail, la maternité représente pour les détenues une identité sociale valorisée et non concurrençable. La légitimité dont est investi le rôle maternel s'accompagne ainsi de puissantes prescriptions, les pratiques de care à destination des enfants, étant pensées comme un devoir incombant aux femmes. Associées à la figure distinctive de la « mauvaise mère » (Cardi, 2008), les détenues en question se voient donc exposées à toutes sortes de brimades. Non seulement leurs pairs sont soumises à une forte pression collective pour ne pas leur parler, mais dans chaque détention se distingue un petit groupe qui s'attache à les persécuter : injures, gifles, humiliation et racket systématique (Joël, 2013). On comprend dès lors pourquoi l'attention que je portai à ces femmes suscita chez mes autres enquêtées une vive indignation. A plusieurs reprises je dus me justifier en expliquant les fondements de la démarche sociologique. Si la mise en avant du principe de neutralité axiologique préconisé par Max Weber me fut utile pour apaiser leur mécontentement, celui-ci ne me fut pas d'une grande aide en revanche pour gérer la sensation de souillure que j'éprouvai à l'écoute de discours révoltants. 


\section{Malaise, souillure...}

La réalisation d'un suivi longitudinal s'avéra précieuse pour le recueil de données. Lors du premier entretien, je ressentis une réserve liée à ce qu'elles me soupçonnaient de détenir déjà une certaine connaissance à leur égard, notamment lorsque leur affaire avait été médiatisée. Face à mon absence de questionnement, elles abordèrent d'ellesmêmes progressivement le sujet. Toutes affirmèrent avoir été victimes d'une injustice, soit qu'elles avaient été contraintes par leur conjoint de participer aux agressions, soit qu'elles ignoraient ce qui se passait et donc avaient été accusées à tort de n'être pas intervenues pour y mettre fin. S'il ne s'agit pas de déterminer qui dit la vérité, l'on constate néanmoins que l'argumentaire de ces femmes se caractérise par une instrumentalisation des stéréotypes de genre à dessin de procéder à un renversement des rôles de victime et de coupable dans la situation. Ainsi, en même temps qu'elles dépeignaient leur conjoint comme un individu dominateur mû par des pulsions sexuelles irrépressibles contre lesquelles elles n'avaient pu protéger leur fille au regard de la crainte qu'il leur inspirait ${ }^{3}$, elles s'efforçaient de me convaincre de l'esprit retors et manipulateur des jeunes victimes en soulignant le but intéressé de leur démarche, voire en les accusant d'attitudes équivoques. L'une des détenues qualifia ainsi ouvertement sa fille de « petite salope », tandis qu'une autre m'assura que son enfant l'avait dénoncée pour toucher de l'argent, n'hésitant pas à se désigner elle-même comme " martyre ". Le recours à cet argumentaire n'est pas anodin puisqu'en mobilisant des stéréotypes de genre, ces femmes produisaient un discours dont elles escomptaient qu'il ferait écho à mes propres représentations et susciterait mon adhésion.

La prolixité dont elles finirent par faire preuve au cours de nos rencontres et les déclarations choquantes auxquelles elles se livrèrent peuvent rétrospectivement s'interpréter comme un signe de réussite : j'étais parvenue à me construire le fructueux statut d'étranger sympathisant, interlocuteur à la fois proche et hors-jeu (Schwartz, 1993 ) ; et à faire de l'entretien une expérience inédite de dévoilement, exempte des coûts symboliques - notamment du jugement moral - qu'induit généralement un tel épanchement. Cela ne me préserva pas pour autant d'une sensation de souillure, les discours recueillis relevant des données anxiogènes évoquées par Georges Devereux (1980). A l'allégresse spontanée que firent naître les confidences succéda en effet un sentiment de malaise diffus. En témoignent mes conversations avec Marguerite, une détenue accusée de complicité d'agressions sexuelles répétées de sa fille par ses fils et son mari. Rassurée par mon attitude, celle-ci s'était lancée dans une véritable diatribe à l'endroit de sa fille. Acquiesçant benoîtement et faisant mine de partager son indignation, je manquai néanmoins m'étrangler lorsqu'elle déclara en riant que celle-ci avait été sodomisée par son père en même temps qu'il lui maintenait la tête dans la cuvette des toilettes. Puisque je m'étais comportée jusque-là de manière à lui démontrer qu'elle ne courait aucun danger à se dévoiler, je me sentis contrainte de m'esclaffer avec elle. Avec les femmes impliquées dans des agressions sexuelles sur mineurs, j’éprouvai ainsi la désagréable impression de trahir leurs victimes.

3 On pensera d'ailleurs à ce que Michelle Martin, l'ex-femme de Marc Dutroux avait affirmé être terrorisée et asservie par son mari pour justifier sa complicité lors de son procès. 
Lorsque le discours des acteurs vient heurter les valeurs profondes fondant la conscience de l'enquêteur, le recueil des données s'accompagne d'un risque de compromission éthique dont Corinne Rostaing (1997) souligne qu'il interroge la relation du chercheur à son objet, son implication et sa distance : cacher ses sentiments ne ressemble-t-il pas à une forme d'acceptation tacite ? La contamination symbolique à laquelle je me trouvais exposée met en exergue les tensions, parfois irréductibles, entre l'identité de chercheur et celle d'individu inscrit dans une structure sociale régie par un système de normes et de valeurs qui, s'ils font partie de ses objets d'étude, ne l'en imprègne pas moins pour autant ${ }^{4}$. Parce qu'elle est noyée dans une peur spécifique entravant la réflexion (Douglas, 1981), la sensation de souillure induit par ailleurs un risque d'occultation. Sur des terrains symboliquement éprouvants, le sociologue doit parfois s'astreindre à approfondir ce qui le heurte, afin de ne pas chercher à maintenir ou à rétablir un ordre social que les données recueillies viennent indubitablement ébranler. Les remarques formulées par Howard Becker à propos des cas inhabituels en sociologie s'avèrent ici particulièrement pertinentes : « L'habitude d'ignorer ce qui est désagréable, déplaisant, de mauvais goût, ou ce qui, d'une manière ou d'une autre, ne doit pas être évoqué, empêche fortement les chercheurs de voir tout l'éventail des cas pertinents et, par conséquent, de s'en servir pour élargir le champ de leurs réflexions » (Becker, 1997 : 33). Parce que la palette de sentiments éprouvés sur le terrain est éminemment complexe, ce qui inspire de la répulsion peut cependant susciter tout à la fois un curieux sentiment d'allégresse.

\section{Mais aussi troublante allégresse pendant les entretiens}

Lorsqu'au détour d'une conversation Noeline évoqua les attouchements qu'elle avait commis sur sa fille handicapée, je ne ressentis spontanément qu'un sentiment de jubilation. J'étais alors au tout début de mon suivi et je voyais dans cette déclaration une piste d'analyse inédite. A la fin de l'entretien cependant, je pris conscience de l'horreur de ma réaction et j'en fus fort troublée : étais-je devenue insensible au point de me réjouir qu'un enfant avait été violenté sexuellement car cela servait mon étude ? Point de passage obligé du travail de terrain, la subjectivité n'est désormais plus honteuse comme en témoigne l'invasion du « je » méthodologique dans les écrits, désormais couramment employé par les sociologues pour partager leurs émotions ressenties sur le terrain (Olivier de Sardan, 2000). Sont ainsi relatés des sentiments de compassion, de joie, de découragement, de malaise, d'indignation ou encore d'affectation, à dessein de se conformer à la démarche critique-analytique (Schwartz, 1993). L'allégresse que peut ressentir le sociologue à l'écoute de récits proprement épouvantables mais féconds pour son étude est en revanche absente de la production scientifique.

$\mathrm{Au}$ cours de ma recherche je fis le constat suivant : chaque fois que j'évoquais les violences sexuelles sur mineur perpétrées par mes enquêtées, mes interlocuteurs, pairs comme profanes, s'attardaient sur les répercussions affectives que de telles déclarations n'avaient pu manquer de me causer, n'hésitant pas à louer mon courage

4 On en trouve une illustration probante dans l'enquête de terrain menée par Philippe Bourgois sur le crack à Harlem, lorsque l'auteur signale que les récits des viols collectifs par ses informateurs le perturbèrent tant qu'ils faillirent remettre en cause sa recherche (Bourgois, 2001). 
et à me revêtir de la peau de « l'ethnologue héros » (Cunha, 2001). Ils se montraient alors réprobateurs si je m'aventurais à leur confesser mon sentiment d'euphorie. Si au départ je me divertis des réactions suscitées par mes déclarations, j'en vins rapidement à éprouver de la honte : étais-je donc la seule à me réjouir d'être confrontée à des atrocités ? Plusieurs discussions avec des collègues m'apprirent qu'il leur arrivait aussi de se délecter d'informations choquantes sur leur propre terrain. Mais pourquoi alors les travaux sociologiques ne portent-ils pas trace d'un tel sentiment ? Cette omission m'amène à formuler une remarque fondamentale : l'on attend certes du sociologue qu'il s'astreigne à une démarche critique-analytique vis-à-vis de son terrain, mais les sentiments qu'il se doit d'expliciter pour mieux contrer les biais qui y sont associés relèvent d'attentes normatives tacites. Plus exactement, l'on escompte moins qu'il fasse preuve de neutralité à propos de choses épouvantables et affligeantes qu'il s'en émeuve d'abord pour être ensuite capable de les traiter.

Les conditions pratiques de la recherche ont longuement été explorées, de même que les attentes du grand public, mais le policing (le maintien de l'ordre professionnel ; Burawoy, 2006) et les attentes entre pairs restent en revanche rarement appréhendés. Pourtant chaque scientifique, pour avoir sa place, est soumis au respect de critères définis par la communauté à laquelle il appartient (Hilgers, 2006). En fait indubitablement partie l'engagement moral pour le «bien » des acteurs sociaux, lequel dessine en creux un modèle normatif de «bon sociologue ». Au cours des années quatre-vingts, les sociologues ont certes opéré des choix de recherche en faveur du quotidien et du vécu, concomitants à un adoucissement de la dimension critique et participative de la sociologie, mais ils ne sont pas départis pour autant d'une forte préoccupation pour le «bien » des acteurs sociaux, dont ils apparaissent aujourd'hui comme les porte-parole de la souffrance et de la plainte qui l'accompagne (Dasseto, 2006). Le sociologue est donc toujours tenu de montrer son engagement dans les problèmes sociaux (Dubet, 2011), quiconque manifestant un déficit d'implication suscitant à son égard un sentiment de trahison (Pinçon, Pinçon-Charlot, 2000).

Le cas particulier de l'allégresse éprouvée sur les terrains difficiles invite ainsi à un niveau plus général à introduire une distinction entre l'engagement idéologique et politique des sociologues tel que celui-ci a participé de divergences - voire de fractures - au sein de la discipline d'une part, et l'engagement moral pour le « bien » des acteurs sociaux tel que celui-là rassemble toujours les sociologues au-delà des clivages épistémologiques, méthodologiques et théoriques d'autre part. Quand bien même est reconnue et félicitée la stricte entreprise de connaissance scientifique, l'on postule toujours plus ou moins - y compris et surtout entre pairs - que le sociologue se préoccupe de la situation des individus auxquels il s'intéresse. Cette consubstantialité de la sociologie et de l'engagement moral pour le « bien » des acteurs sociaux a pour conséquence qu'il est sociologiquement incorrect d'évoquer sa jubilation lors du recueil de faits terribles et attristants.

S'il évite de donner une image de laideur de ses enquêtés (Bourgois, 2001), le sociologue s'efforce donc de ne pas renvoyer non plus une image défavorable de lui-même, ce qui s'observe avec acuité sur les terrains difficiles où l'allégresse parfois éprouvée lors du recueil de données disparaît dans la production scientifique derrière la mise en avant de sentiments plus légitimes. A l'instar d'autres catégories professionnelles, l'adoption d'une attitude distanciée par rapport à des valeurs 
intangibles ou à des choses qui suscitent des attachements inébranlables, et l'emploi de formules choquantes pour parler des acteurs et de leurs problèmes, marquent pourtant bien le rassemblement des sociologues comme groupe professionnel établi (Hughes, 1996). Qu'en est-il alors de la diffusion des résultats issus de ces terrains?

\section{Evoquer l'indicible}

Dans les travaux sociologiques contemporains, il est courant de s'appesantir sur les obstacles rencontrés pendant l'enquête. Sont en revanche occultées les difficultés relatives à la diffusion des résultats dans les publications et les communications. En ce qui concerne les violences sexuelles sur mineurs commises par des femmes, la problématique a tout à la fois l'avantage et l'inconvénient d'être de l'ordre du sensationnel. Un avantage d'abord car en provoquant une forte impression sur les lecteurs ou sur les auditeurs, elle suscite indéniablement leur intérêt. Lors de mes communications, je dois ainsi à Marguerite et Noeline d'avoir introduit mes analyses dans un silence des plus attentifs. Si le sensationnel captive, il présente néanmoins l'inconvénient d'éclipser d'autres savoirs moins inattendus, voire de jouer contre l'entreprise sociologique en venant compliquer l'effort d'objectivation. J'entends par là que l'objet d'étude tend à réactiver le registre « chaud » de la révolte, de la dénonciation ou de l'indignation, là où il importe précisément de faire preuve de froideur et de distance (Pollak, 1990). Au cours des discussions suivant mes interventions, je constatai que mes auditeurs écœurés avaient davantage retenu que les prisons comptaient des mères ayant violente sexuellement et prostitué leurs enfants que les processus sociaux à l'œuvre dans ce type d'infraction.

Je redoublai ainsi de vigilance quant à la terminologie employée, ce qui posa la question de savoir si je devais réutiliser les termes parfois choquants de mes enquêtées (notamment les injures à destination des enfants). Afin de conserver l'avantage du sensationnel tout en se préservant de ses inconvénients, il me parut fécond de les diluer dans l'analyse en veillant à les articuler étroitement aux théories présentées. La tentation fut grande de les utiliser comme des projecteurs sur la réalité observée, mais je les maniai avec précaution dans la mesure où je savais qu'ils susciteraient spontanément de fortes réactions affectives. Dans mes communications par exemple, je ne citai le qualificatif de " petite salope » employé par Marguerite qu'au moment où j'exposai le processus d'instrumentalisation des stéréotypes de genre. J'avais en effet remarqué que si je ne veillais pas à cette articulation, l'indignation de mes auditeurs entravait leur appréciation globale du phénomène en limitant leur attention à sa dimension anecdotique choquante.

Le sociologue qui enquête sur des terrains sensibles court par ailleurs le risque en particulier auprès d'un public profane - d'être tenu pour défenseur d'une position amorale, comme si son intérêt relevait d'une volonté de défendre des convictions personnelles douteuses par le truchement d'un travail d'investigation. Howard Becker (1985) faisait remarquer que par le fait même d'exposer les rationalisations et les justifications auxquelles ont recours les acteurs, le chercheur parait accepter celles-ci ; et cela se vérifie notamment pour les faits de violence sexuelle sur mineurs. Dès lors que je livrais mes analyses, mes interlocuteurs ne manquaient pas de m'interroger sur mon opinion personnelle à propos de leurs auteures : il leur était manifestement 
insupportable que je m'en tienne à un discours rationnel dénué de toute condamnation. L'attitude d'objectivité et le détachement à l'égard de certains objets pouvant être tenus pour des déviations menaçantes pour l'ordre social (Hughes, 1996), il existe des attentes normatives quant à la façon dont le sociologue les évoque dans la sphère publique : on attend de lui un positionnement moral venant ratifier les normes fondatrices du lien social. Cela pose finalement la question de la diffusion des savoirs sociologiques. Dans un contexte où la parole du « sociologue-expert » est de plus en plus sollicitée (Mattart, 2006) et où les chercheurs sont invités à dépasser la seule sphère académique pour s'ouvrir à de nouveaux supports et à des publics élargis (Lemieux et al., 2010), tous les savoirs peuvent-ils être exportés? S'ils doivent subir une stricte mise en forme pour être rendus dicibles dans l'espace public, cela signifie-t-il que l'entreprise de connaissance ne peut véritablement être comprise et agréée en-dehors du milieu scientifique ?

Les attentes normatives sont exprimées de manière explicite par les profanes, mais elles existent aussi en filigrane chez les pairs. Rappelons ici les déclarations de Michel Pinçon et Monique Pinçon-Charlot (2000) quant aux soupçons de leurs collègues visà-vis du caractère empathique de leurs relations avec leurs enquêtés appartenant à la grande bourgeoisie : on les suspectait d'adhérer aux pratiques d'utilisation du système au profit des plus nantis. Un autre aspect vient compliquer la diffusion des résultats au sein de la sphère scientifique. A partir de mon étude sur la sexualité en prison de femmes, je parvins à publier plusieurs articles. Or tous ceux traitant des violences sexuelles sur mineurs furent refusés, parfois même avant d'être évalués par le comité de lecture. La recherche par mot-clé sur des moteurs comme Jstor met bien en évidence les processus de légitimation ou de délégitimation en jeu dans l'activité de publication scientifique, qui ne viennent finalement que rejouer ceux du champ médiatique. Al'autre extrémité de ce continuum de légitimité, la problématique de la maternité en prison fait ainsi complètement écran, au travers de l'importance écrasante des publications qui y sont consacrées, à celle des violences sexuelles sur mineurs commises par des femme ; cette relégation n'étant qu'un écho de la disparité d'intérêt manifesté par les journalistes : sollicitée une bonne trentaine de fois pour être interviewée sur la première problématique depuis ma soutenance de thèse, je ne le fus pas une seule fois sur la seconde.

\section{Conclusion}

Parce que les violences sexuelles sur mineur commises par des femmes font partie de ces terrains minés qui exigent une conscience aiguë de la part de l'enquêteur vis-à-vis de son travail (Arrif, 2001), l'article a permis d'examiner quatre séries de difficultés auxquelles risque de se trouver confronté le sociologue enquêtant en terrain dit difficile : accéder aux acteurs, gérer la sensation de souillure, se confronter à la honte du sentiment d'allégresse et diffuser ses résultats. Si certains de ces obstacles peuvent finalement se retrouver dans toute enquête et s'observent avant tout ici avec davantage de force, la spécificité de quelques autres éclaire en miroir le système de normes tel qu'il sous-tend les pratiques et les représentations des sociologues à leur métier et tel qu'il concourt à définir les attentes élaborées à leur endroit dans la sphère profane comme scientifique. 
Qu'ils amènent le sociologue à perfectionner ses techniques d'enquête, à questionner son rapport aux valeurs, à prendre conscience de ses limites ou à s'interroger sur la diffusion des connaissances qu'il produit, les terrains difficiles invitent à réfléchir sur les contradictions et les points de rencontre entre la posture de scientifique et celle d'acteur social. Jean-Pierre Olivier de Sardan (2000) faisait remarquer que trop de méthodologie tue la méthodologie, aussi, s'il serait vain d'analyser à outrance les us et coutumes de nos pairs, il convient cependant de garder à l'esprit que la pratique sociologique n'en demeure pas moins une activité sociale comme une autre.

\section{Références citées}

ArriF, Abdelmajid, 2001. «Fragments d'une enquête dans un bidonville de Casablanca », Ethnologie française, 31 (1), pp. 29-39.

BECKER, Howard, 1997. « La prise en compte de cas inhabituels dans l'analyse sociologique : les conseils de Hughes ». Sociétés contemporaines, 27, pp. 29-37.

Benguigui, Georges, Antoinette Chauvenet et Françoise Orlic, 1994. Le monde des surveillants de prison. Paris : PUF.

Bızeul, Daniel, 1998. «Le récit des conditions d'enquête : exploiter l'information en connaissance de cause », Revue française de sociologie, 39 (4), pp. 751-787.

Bourgols, Philippe, 2001. En quête de respect : le crack à New York. Paris : Seuil.

BRIE, Guillaume, 2014. Des pédophiles derrière les barreaux. Comment traiter un crime absolu ? Paris : l'Harmattan.

Burawoy, Michael, 2006. « Pour la sociologie publique », Socio-logos, 1.

CARDI, Coline, 2008. « La déviance des femmes. Délinquantes et mauvaises mères : entre prison, justice et travail social », thèse sous la direction scientifique de Numa Murard. Paris : Université Denis Diderot.

CARdi, Coline et Geneviève Pruvost, 2011. « La violence des femmes : occultations et mises en récit », Champ Pénal, Penal Field, 8.

Cliquennois, Gaëtan, 2006. « Problèmes méthodologiques inhérents à une recherche sociologique qualitative menée sur les politiques carcérales belges et françaises », Socio-logos, 1.

Cunha, Manuela Ivone, 2001. « Trajets et dérives autour d'une prison de femmes », Ethnologie française, 31 (1), pp. 81-87.

DAsseto, Felice, 2006. « Sociologies et sociétés : une articulation à repenser ? », Recherches sociologiques et anthropologiques, 37 (1), pp. 7-26.

Devereux, Georges, 1980. De l'angoisse à la méthode dans les sciences du comportement. Paris : Flammarion.

Douglas, Mary, 1981 [1971]. De la souillure. Essai sur les notions de pollution et de tabou. Paris : Maspero.

DuBEt, François, 2011. A quoi sert vraiment un sociologue? Paris : Armand Colin.

Faure, Michaël, Lilian Mathieu et Daniel Welzer-Lang, 1996. Sexualité et violences en prison : ces abus $q u$ 'on dit sexuels. Lyon : Aléas.

Gaillard, Arnaud, 2009. Sexualité et prison. Désert affectif et désirs sous contrainte. Paris : Max Milo.

Giuliani, Fabienne, 2011. « L’impossible crime », Champ Pénal, Penal Field, 8.

Hilgers, Mathieu, 2006. « La responsabilité sociologique : retour sur l'entreprise critique de Pierre Bourdieu », Recherches sociologiques et anthropologiques, 37 (1), pp. 43-63.

Hughes, Everett, 1996. Le regard sociologique. Paris : EHESS. 
JoËL, Myriam, 2013. « Coûts et bénéfices de l'activité homosexuelle dans l'espace de la détention féminine», Ethnologie Française, 43 (3), pp. 469-476.

-, 2014. « Conduites sexualisées et pouvoir dans les prisons de femmes », Hermès, 69, pp. 65-70.

MatTART, Christophe, 2006. « Le “sociologue-expert” à la télévision : un sens pour la posture sociologique ? », Recherches sociologiques et anthropologiques, 37 (1), pp. 85-103.

Melcher, Claudia, 2002. «"A mon tour d'être le monstre...”. Violences sexuelles infligées par des femmes », Revue internationale de criminologie et de police technique et scientifique, 55 (4), pp. 410-431.

Olivier de SARDAN, Jean-Pierre, 2000. « Le “je” méthodologique. Implication et explicitation dans l'enquête de terrain », Revue française de sociologie, 41 (3), pp. 147-445.

Pinçon, Michel et Monique Pinçon-Charlot, 2000. « Enquête en grande bourgeoisie. L'implication du chercheur et les difficultés de l'engagement comme trahison des enquêtés » in Philippe Fritsch (éd.), Implication et engagement. Hommage à Philippe Lucas, pp. 135-139. Lyon : Presses universitaires de Lyon.

Pollak Michael, 1990. L'expérience concentrationnaire. Paris : Métailié.

RostaIng, Corinne, 1997. La relation carcérale. Identités et rapports sociaux dans les prisons de femmes. Paris : PUF.

Schwartz, Olivier, 1993. «L'empirisme irréductible », in Nels Anderson, Le Hobo. Sociologie du sans-abri, pp. 265-308. Paris : Nathan. 